\title{
6-Amino-3-Methyl-4-(2-nitrophenyl)-1,4-Dihydropyrano[2,3-c] Pyrazole-5-Carbonitrile Shows Antihypertensive and Vasorelaxant Action via Calcium Channel Blockade
}

\author{
Authors \\ Samuel Estrada-Soto1, Priscila Rendón-Vallejo ${ }^{1{ }^{*}}$, Rafael Villalobos-Molina ${ }^{2}$, César Millán-Pacheco', \\ Miguel A. Vázquez ${ }^{3}$, Fernando Hernández-Borja ${ }^{3}$, Emanuel Hernández-Núñez ${ }^{4}$
}

\section{Affiliations}

1 Facultad de Farmacia, Universidad Autónoma del Estado de Morelos, Cuernavaca, Morelos, México

2 Unidad de Biomedicina, Facultad de Estudios SuperioresIztacala, Universidad Nacional Autónoma de México, Tlalnepantla, Estado de México, México

3 Departamento de Química, División de Ciencias Naturales y Exactas, Universidad de Guanajuato, Guanajuato, México

4 Cátedra CONACyT, Departamento de Recursos del Mar, Centro de Investigación y de Estudios Avanzados del IPN, Unidad Mérida, Yucatán, Mexico

\section{Key words}

4H-pyran derivative, antihypertensive, calcium channel

blockade, dihydropyridines, nifedipine, vasorelaxant

received 19.07 .2021

revised 20.08.2021

accepted 24.08.2021

published online 18.10 .2021

Bibliography

Drug Res 2022; 72: 53-60

DOI 10.1055/a-1616-0156

ISSN 2194-9379

(C) 2021. Thieme. All rights reserved.

Georg Thieme Verlag, Rüdigerstraße 14,

70469 Stuttgart, Germany

Correspondence

Dr. Samuel Estrada-Soto

Facultad de Farmacia, Universidad Autónoma del Estado de Morelos,

Av. Universidad 1001

Cuernavaca

62116 Morelos

México

Tel.: + 5277732970 89, Fax: + 527773297089

enoch@uaem.mx
\# Supplementary material is available under https://doi.org/10.1055/a-1616-0156

\begin{abstract}
Several $4 H$-pyran derivatives were designed and synthesized previously as vasorelaxant agents for potential antihypertensive drugs. In this context, the objective of the present investigation was to determine the functional mechanism of vasorelaxant action of 6-amino3-methyl-4-(2-nitrophenyl)-1,4-dihydropyrano[2,3-c]pyrazole5-carbonitrile (1) and its in vivo antihypertensive effect. Thus, compound 1 showed significant vasorelaxant action on isolated aorta rat rings pre-contracted with serotonin or noradrenaline, and the effect was not endothelium-dependent. Compound 1 induced a significant relaxant effect when aortic rings were contracted with $\mathrm{KCl}(80 \mathrm{mM})$, indicating that the main mechanism of action is related to L-type calcium channel blockade. Last was corroborated since compound 1 induced a significant concentration-dependent lowering of contraction provoked by cumulative $\mathrm{CaCl}_{2}$ adding. Moreover, compound 1 was capable to block the contraction induced by FPL 64176, a specific L-type calcium channel agonist, in a concentrationdependent manner. On the other hand, docking studies revealed that compound $\mathbf{1}$ interacts on two possible sites of the L-type calcium channel and it had better affinity energy $(-7.80+/-0.00 \mathrm{kcal} / \mathrm{mol}$ on the best poses) than nifedipine $(-6.86+/-0.14 \mathrm{kcal} / \mathrm{mol})$. Finally, compound $1(50 \mathrm{mg} / \mathrm{kg})$ showed significant antihypertensive activity, lowering the systolic and diastolic blood pressure on spontaneously hypertensive rats (SHR) without modifying heart rate.
\end{abstract}

* "Taken in part from the PhD thesis of P. Rendón-Vallejo. 


\section{Introduction}

Cardiovascular diseases (CVDs) are the main cause of death globally; an estimated of 17.9 million people died from CVDs in 2016, representing $31 \%$ of all global deaths. Of these deaths, $85 \%$ were due to heart attack and stroke, and the main risk factor for both is hypertension, a complex and multifactorial disorder in which persistently high blood pressure (HBP) can damage blood vessels and heart. Also, HBP accounts for 1.13 billion people around the world [1]. For the treatment of this chronic non-communicable disease a variety of drugs exist, classified by their mechanism of action as angiotensin II antagonists, adrenergic antagonists, calcium channel blockers, direct vasodilators, diuretics, and angiotensin converting enzyme inhibitors [2]; however, these drugs can cause tolerance and side effects after a period of use. Therefore, several research groups currently focus on discover and develop new antihypertensive agents to counteract those effects following new strategies, such as the rationalized structural modification of drugs with potent therapeutic activity, with specific mechanism of action or by the multitarget actions. In this regard, compounds derived from dihydropyridines as nifedipine, a powerful calcium channel blocker used for the treatment of HBP and angina pectoris [3], are useful for the design and synthesis of novel calcium channel blockers with significant vasorelaxant action used for developing new antihypertensive drugs [4]. In this context, previously we obtained new isosteric $4 \mathrm{H}$-pyrane derivatives of dihydropyridines, which showed potent vasorelaxation [4], being 6-amino-3-methyl-4-(2nitrophenyl)-1,4-dihydropyrano[2,3-c]pyrazole-5-carbonitrile (1) one of the most active compounds of the series ( $\mathbf{F i g}$. 1). Thus, the aim of this work was to seek the vasorelaxant mode of action of compound $\mathbf{1}$ on isolated rat aorta and in in silico studies, as well as testing in vivo its acute antihypertensive and toxicological activities in murine models.

\section{Materials and Methods}

\section{Chemical and Drugs}

Compound 1 was obtained previously [4]. Carbamoylcholine chloride (carbachol), ( \pm )-noradrenaline bitartrate hydrate (NA), serotonin chloride $(5-\mathrm{HT})$, nifedipine, $1 \mathrm{H}$-[1, 2, 4]oxadiazolo[4,3-a]quinoxalin-1-one (ODQ), glibenclamide, tetraethylammonium chloride (TEA), FPL 64176, components of physiological solution, and dimethylsulfoxide (DMSO) were purchased from Sigma-Aldrich Co. (St. Louis, MO, USA). For ex vivo and in vivo experiments, compound 1 was dissolved in distilled water and DMSO (1\% and $10 \% \mathrm{v} / \mathrm{v}$, respectively). Preliminary experiments showed that DMSO had no effect on tension development on isolated aorta and on the blood pressure of SHR.

\section{Animals}

Male Wistar rats (3 months-old), spontaneously hypertensive (SHR) rats ( 8 months-old), and CD1 mice (3 months-old), were used and maintained under standard laboratory conditions with free access to food and water. All animal practices were guided followed our Federal Regulations for Animal Experimentation and Care (SAGARPA, NOM-062-ZOO-1999, Mexico), and approved by the Institu-

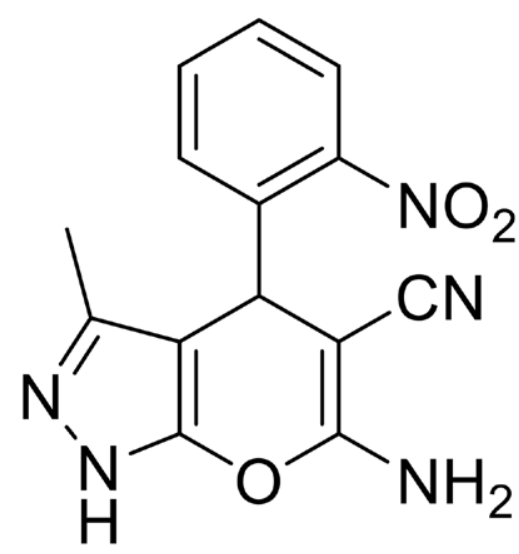

Fig. 1 6-amino-3-methyl-4-(2-nitrophenyl)-1,4dihydropyrano[2,3-c]pyrazole-5-carbonitrile (1).

tional Animal Care and Use Committee (Protocol 1368, F.E.S. Iztacala). Wistar rats and mice were provided by F.E.S. Iztacala, and SHR were supplied by Cell Physiology Institute animal facilities, from Universidad Nacional Autónoma de México, respectively.

\section{Functional studies}

Wistar rats (250-350 g body weight) were killed by exposure to ethyl ether. The thoracic aorta was dissected out, cleaned of adhering connective tissue, and cut into $4-5 \mathrm{~mm}$ length rings. Some rings were denuded of endothelium. Then, aortic rings were hooked to the bottom of the chamber and to a force transducer under an optimal tension of $3 \mathrm{~g}$, in $10 \mathrm{~mL}$ organ baths containing warmed $\left(37^{\circ} \mathrm{C}\right)$ and oxygenated $\left(\mathrm{O}_{2} / \mathrm{CO}_{2}, 95: 5\right)$ Krebs solution (composition in $\mathrm{mM}: \mathrm{NaCl}, 118 ; \mathrm{KCl}, 4.7 ; \mathrm{CaCl}_{2}$, 2.5; $\mathrm{MgSO}_{4}, 1.2$; $\mathrm{KH}_{2} \mathrm{PO}_{4}, 1.2 ; \mathrm{NaHCO}_{3}, 25.0$; EDTA, 0.026, and glucose, 11.1, $\mathrm{pH}$ 7.4). Changes in tension were recorded by Grass FT03 force transducers (Astromed, West Warwick, RI, USA), connected to a MP100 Manager Biopac System polygraph (Biopac Instruments, Santa Barbara, CA, USA). After the stabilization period, the vessels were stimulated with noradrenaline (NA, $0.1 \mu \mathrm{M}$ ) during $10 \mathrm{~min}$, and then washed with fresh Krebs solution. The stimulation was done three times at 30 min intervals before starting the experiments. The absence of endothelium was confirmed by the lack of the relaxant response induced by carbachol $(1 \mu \mathrm{M})$ in the last stimulation [5-7].

\section{Determination of functional mode of action}

Effect on contractions induced by NA $(0.1 \mu \mathrm{M})$ or $5-\mathrm{HT}$ $(0.1 \mu \mathrm{M})$

The aortic rings with and without endothelium were precontracted with $\mathrm{NA}(0.1 \mu \mathrm{M})$ or $5-\mathrm{HT}(0.1 \mu \mathrm{M})$. Once a plateau was reached, concentration-response curves of the relaxation induced by 1 was obtained by adding cumulative concentrations to the bath [6-8].

Effect on the cumulative contractions induced by NA

Endothelium-denuded aortic rings were incubated with 98, 55, 175 and $306 \mu \mathrm{M}$ of 1 during $15 \mathrm{~min}$; then NA was added at different concentrations $(0.1-10 \mu \mathrm{M})$. Finally, the contractile effect induced by 
NA was compared in the absence (control group) and in the presence of $\mathbf{1}[8,9]$.

Effect on the contractions induced by potassium chloride $(\mathrm{KCl})$

$\mathrm{KCl} 80 \mathrm{mM}$ was added to the aortic rings without endothelium to contract them. After, cumulative concentration-response curves of 1 -induced relaxation were obtained $[6,7,10]$.

Effect on extracellular $\mathrm{Ca}^{2+}$ induced contractions activated by $\mathrm{KCl}$

To observe if the inhibition of extracellular $\mathrm{Ca}^{2+}$ influx was involved in $\mathbf{1}$-induced relaxation, the tests were conducted in $\mathrm{Ca}^{2+}$-free Krebs solution. Endothelium-denuded aortic rings were washed with $\mathrm{Ca}^{2+}$-free solution (during $20 \mathrm{~min}$ ), then rinsed with $\mathrm{Ca}^{2+}$-free solution containing $\mathrm{KCl}(80 \mathrm{mM})$. After that, the cumulative concentration-response curves for $\mathrm{CaCl}_{2}(80 \mu \mathrm{M}$ to $27 \mathrm{mM})$ were attained in the absence of $\mathbf{1}$ (control group), or after 15 min of incubation with the test sample ( 55 and $98 \mu \mathrm{M})$. Finally, the contractile effect induced by $\mathrm{CaCl}_{2}$ was compared in the absence (control group) and in the presence of test compound $[7,9,11]$.

Involvement of L-Type calcium channels in the vascular action of $\mathbf{1}$

To confirm L-type calcium channel participation as the main target of the vascular action of $\mathbf{1}$, endothelium-denuded aortic rings were incubated with 31 and $98 \mu \mathrm{M}$ of 1 for $15 \mathrm{~min}$. Then, $3.16 \mu \mathrm{M} \mathrm{FPL}$ 64176 (L-type channel agonist) was added. The maximal tension induced by FPL 64176 in the control group (without test compound) was considered as $100 \%$ contraction. As a final point, the contractile effect induced by FPL 64176 was compared in the absence and presence of $\mathbf{1}[7,9]$.

Role of $\mathrm{K}^{+}$channel in the relaxation induced by $\mathbf{1}$

To observe if $\mathrm{K}^{+}$channels are involved in $\mathbf{1}$-induced relaxation, rat aortic rings without endothelium were preincubated with the $\mathrm{K}^{+}$ channel blocker TEA $(5 \mathrm{mM}), 15$ min before NA $(0.1 \mu \mathrm{M})$ was placed. Subsequently, 1 was cumulatively added. After all, 1 -induced vasorelaxation was compared in the absence (control group) and in the presence of $\mathrm{K}^{+}$channel blockers $[7,9,10]$.

\section{In vivo studies}

Determination of antihypertensive action of $\mathbf{1}$

Antihypertensive activity study of 1 was conducted in SHR [(250$300 \mathrm{~g} ; 408 \pm 6.3 \mathrm{bpm}$ for hearth rate (HR); $183 \pm 5.6$ and $138 \pm 3.5 \mathrm{mmHg}$ for systolic blood pressure (SBP) and diastolic blood pressure (DBP), respectively)]. Rats were allocated into three groups (six animals each): Control rats [DMSO 10\% (group 1)], treated rats [Compound $\mathbf{1}(50 \mathrm{mg} / \mathrm{kg})$, group 2] and positive control group [nifedipine $(50 \mathrm{mg} / \mathrm{kg})$, group 3]. Determination of blood pressure and heart rate were documented before and after the intragastric treatment of test samples at $0,1,3,5$ and $7 \mathrm{~h}$ by a tail cuff method using a LE 5007 automatic blood pressure recorder (Letica1, PanLab, Barcelona, Spain). Percent decrease in HR, SBP, and DBP were calculated [12].

\section{Acute toxicity in mice}

This trial was carried out following the suggestions of the Organization for Economic Co-operation and Development (OECD) Guidelines 423, for an in vivo oral toxicity model with some modifications. For this study, CD1 male mice were used, which were weighed and divided into 4 groups of 3 animals each. Subsequently, a single dose of 1 was administered intragastrically to each group, according to the doses described in the OECD protocol $(5,50,500$ and $2000 \mathrm{mg}$ / kg body weight). It is important to mention that $\mathbf{1}$ was dissolved in $10 \%$ DMSO. On the other hand, the mortality rate and toxic reactions observed at the beginning and during the experiment were monitored during the test up to 24 hours post-dose [12, 13].

\section{Molecular docking}

Molecular docking of compound $\mathbf{1}$ and nifedipine were done on voltage-dependent L-type calcium channel structures obtained from electron microscopy. Voltage-dependent L-type calcium channel of Oryctolagus cuniculus were used (PDB ID: 6JPA, 6JPB, 6JP5 and 6JP8) [14, 15]. These structures showed the L-type calcium channel with different ligands with partially overlapped binding sites (see Supporting information). Even when this study was done with nifedipine and compound $\mathbf{1}$, we explored the possibility that compound $\mathbf{1}$ may bind to other binding site of the same calcium channel by docking it on all four structures. All structures were aligned and a grid box larger enough to fit all experimental ligands were used. A grid box of $20 \times 20 \times 20 \AA^{3}$ was used for all structures. Grid box center after the structural alignment was on (163.78, $187.11,172.58)$ coordinates. 100 independent docking experiments were realized on each structure with Autodock Vina [16]. Original ligand (nifedipine for 6JP5) and compound $\mathbf{1}$ were tested. Images and interaction maps were done using Maestro 2021-2 from Schrodinger [17] and VMD 1.9.4a51 [18].

\section{Statistical analysis}

Data were expressed as means \pm standard error of the mean (SEM). Statistical analysis was conducted using one-way ANOVA, followed by Tukey's post hoc test. A $p$ value of $<0.05$ was considered to be significant. Concentration-response curves were plotted, and the obtained experimental data were adjusted by a nonlinear curves fitting program (ORIGIN 6.0).

\section{Results and Discussion}

\section{Ex vivo studies}

Compound $\mathbf{1}$ previously designed and synthesized showed significant vasorelaxant action, in a concentration-dependent and endothelium-independent manners, against contraction induced by noradrenaline $(0.1 \mu \mathrm{M})$, which indicated that the mechanism of functional action should be evaluated in endothelium-denuded rat aorta rings $[7,8]$. As it is known, an augment of intracellular calcium mediated by NA stimulus has a functional role for the contraction in vascular smooth muscle, therefore a calcium entry blocker induces vasorelaxation, and in consequence a potent antihypertensive effect is produced. Hence, when we found that $\mathbf{1}$ inhibited the contractile effect induced by NA or serotonin ( $\triangleright$ Figs. $2 a$ and 2b) in endothelium-denuded aortic rings, suggested that its 

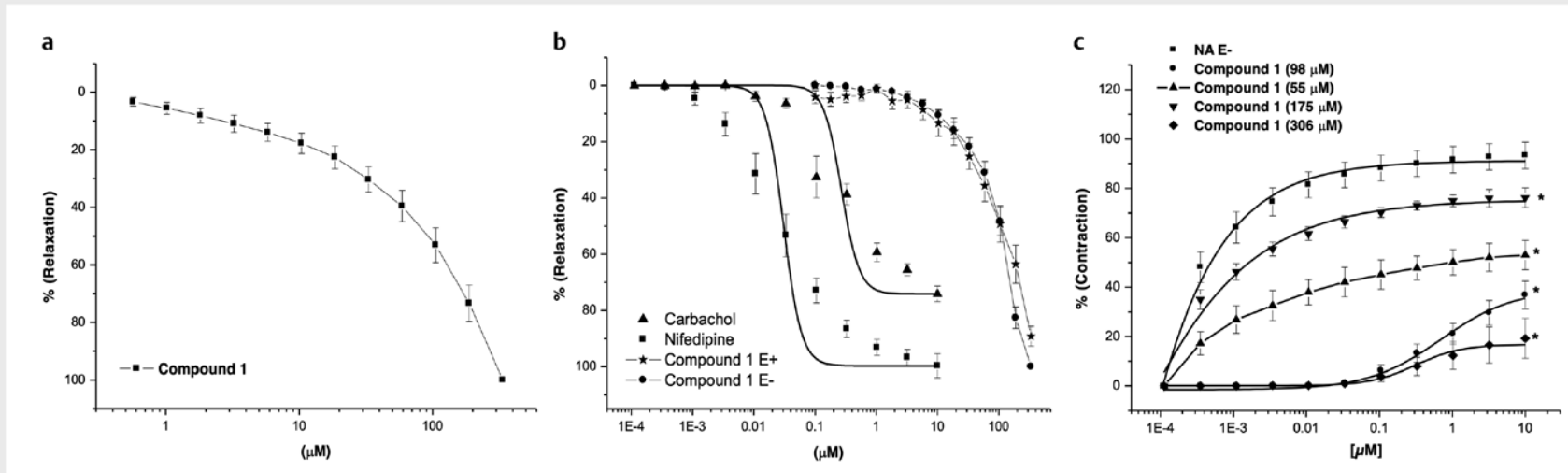

Fig. 2 Vasorelaxant action of compound 1 on a) serotonin, b) noradrenaline and c) on the NA-contraction $(1.01-521 \mu \mathrm{M})$ in rat endotheliumfree aorta rings ( ${ }^{*} p<0.05$ vs. control).

functional mode of action is not related with a serotoninergic or adrenergic competitive antagonism, rather to some other common process involved in the interruption of contraction due to neurotransmitters $[6,19]$. To confirm this, the effect of compound 1 at different concentrations $(55,75,98$ and $306 \mu \mathrm{M})$ on concentrationdependent contraction induced by NA $(1.01-521 \mu \mathrm{M})$ was evaluated. As observed in $\mathbf{F i g . ~} \mathbf{2 c}$, the inhibition of contraction was significant, showing a constant decrease in the efficacy (maximum effect) and potency (a displacement to the right of the contraction curves), in the presence of different concentrations of $\mathbf{1}$, inducing vessel relaxation in a concentration-dependent manner. Based on the effect showed in $\mathbf{F i g . ~} \mathbf{2 c}$, the functional mechanism of action could be related with a non-competitive antagonism, which suggests that compound $\mathbf{1}$-induced relaxation is linked with a common step interaction [18]. This could be related with an interference of the calcium entry into the aortic smooth muscle cells $[6,19]$. Thus, to corroborate latter hypothesis, it was designed next experiments: aortic rings were contracted with $\mathrm{KCl} 80 \mathrm{mM}$, that induced a membrane depolarization provoking an entry of extracellular $\mathrm{Ca}^{2+}$ through L-type calcium channel, which augment the intracellular $\left[\mathrm{Ca}^{2+}\right]$ that finally induced contraction [20]. Thus, compound $\mathbf{1}$ produced significant vasorelaxation on contraction provoked by $\mathrm{KCl}$, in a concentration-dependent fashion in aortic rings without endothelium ( $\vee$ Fig. 3a); however, it was less potent than nifedipine, but with the same efficacy. According to the data obtained, compound 1 is able to block L-type calcium channel [21, 22]. Therefore, the second experiment consisted of pre-incubate the aortic rings with compound $\mathbf{1}$ in the presence of physiological solution without calcium, and after that cumulative concentration-response curves to $\mathrm{CaCl}_{2}$ were obtained. As shown in $\mathbf{F i g}$. $\mathbf{3 b}$, compound $\mathbf{1}$ (55 and $98 \mu \mathrm{M}$ ) significantly reduced contraction induced by $\mathrm{CaCl}_{2}$ in a concentration-dependent manner causing that, at the concentration of $98 \mu \mathrm{M}$, contraction was totally abolished. This supports the concept that test sample 1 possesses $\mathrm{Ca}^{2+}$ entry-blocking activity, since it was developed and synthesized as isosteric derivative of dihydropyridines (DHP) such as nifedipine [4, 21, 23], a potent calcium channel blocker, which is widely used as an antihypertensive agent [24]. Finally, a third experiment was conducted to clearly establish the functional mechanism of action of $\mathbf{1}$, which consisted in the use of a direct L-type calcium channel agonist, FPL-64176
(FPL). The benzoylpyrrole derivative FPL increased L-type Ca ${ }^{2+}$ channel current magnitude opening that induce a potent contraction in tissue [9, 25, 26]. Accordingly, the L-type (voltage-gated) $\mathrm{Ca}^{2+}$ channel blockade participation in the vasorelaxant effect of 1 was confirmed, since it significantly diminished contractions induced by FPL $(3.16 \mu \mathrm{M})$, in a concentration-dependent manner ( $\triangleright$ Fig. 4). Furthermore, with the three experiments carried out we established that compound $\mathbf{1}$ produced significant vasorelaxation via L-type calcium channel blockade, which verified that the design and synthesis of isosteric analogues of DHP can function as potential antihypertensive drugs, with the aforementioned mechanism of action.

On the other hand, to validate or discard if the effect of the assayed compound is related to the opening of $\mathrm{K}^{+}$channels, which induces hyperpolarization of the membrane potential in vascular smooth muscle cells, aortic rings without endothelium were incubated with TEA (non-specific $\mathrm{K}^{+}$channel blocker) [9]; however, the experimental data showed no difference with respect to the control curve ( $\triangleright$ Fig. 5), therefore, the vasorelaxant mechanism $\mathbf{1}$ is not related to the opening of the voltage-dependent $\mathrm{K}^{+}$channels.

\section{Docking studies}

Compound $\mathbf{1}$ and nifedipine were docked on voltage dependent L-type calcium channel of Oryctolagus cuniculus. Sequence alignment of Oryctolagus cuniculus and Rattus norvegicus showed a $92.1 \%$ of identity, allowing to use those structures of $O$. cuniculus as representatives of L-type calcium channels present on Wistar rats. Even more, those residues that interact with ligands on L-type calcium channels used on this study are $100 \%$ conserved on both organisms (Supporting information). O. cuniculus structures showed that verapamil and diltiazem bound to the middle part of the channel, right below the channel filter; meanwhile, nifedipine and $k 8644$ are bound on the inner part of the channel below of one of the channel filter helixes.

In this work, we tested compound $\mathbf{1}$ and nifedipine affinities on four structures of L-type calcium channel using molecular docking [15], where 100 independent experiments were conducted [16].

Molecular docking grid box was large enough that it includes all four binding sites found on the experimental structures. As noted, nifedipine was found on $54 \%$ on its original binding site. These re- 

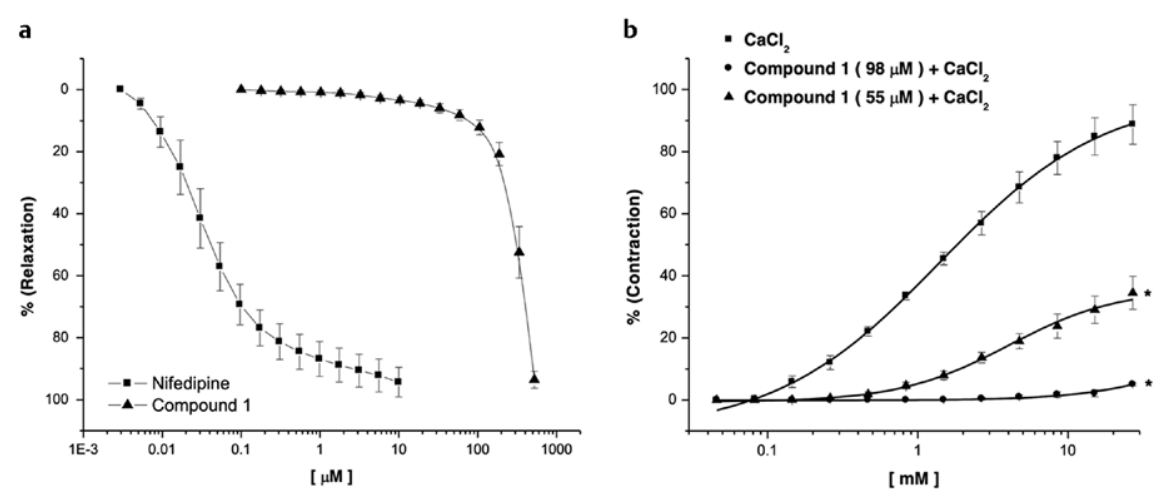

- Fig. 3 Effect of compound 1 on a) $\mathrm{KCl}$-induced contraction $(80 \mathrm{mM})$ and $\mathbf{b})$ effect of compound $\mathbf{1}(55$ and $98 \mu \mathrm{M})$ on $\mathrm{CaCl}_{2}$-induced contraction $(80 \mu \mathrm{M}$ to $27 \mathrm{mM})$ in aortic rings without endothelium ( ${ }^{*} \mathrm{p}<0.05 \mathrm{vs}$. control).

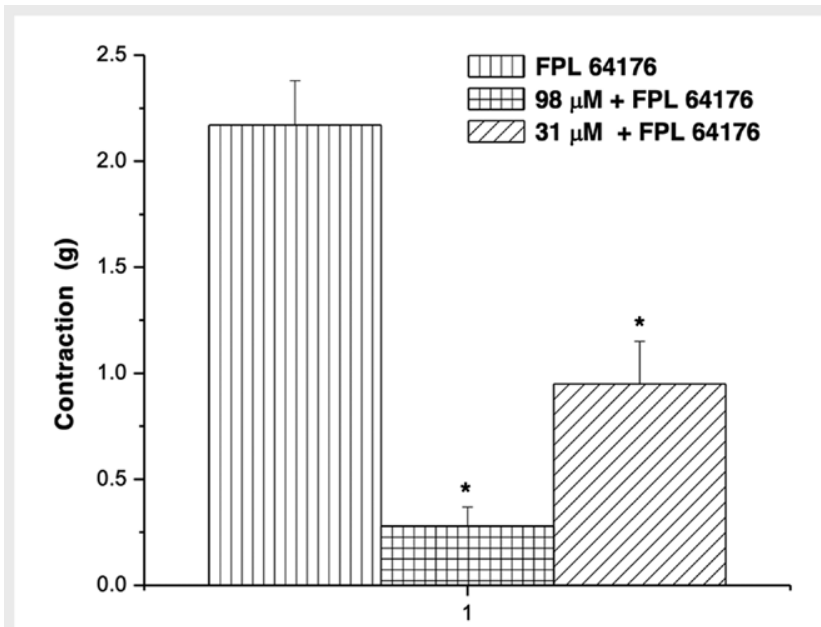

- Fig. 4 Effect of compound 1 ( 31 and $98 \mu \mathrm{M}$ ) on FPL-64176 $(3.16 \mu \mathrm{M})$ induced contraction in rat endothelium-denuded aorta rings ( ${ }^{*} \mathrm{p}<0.05$ vs. control).

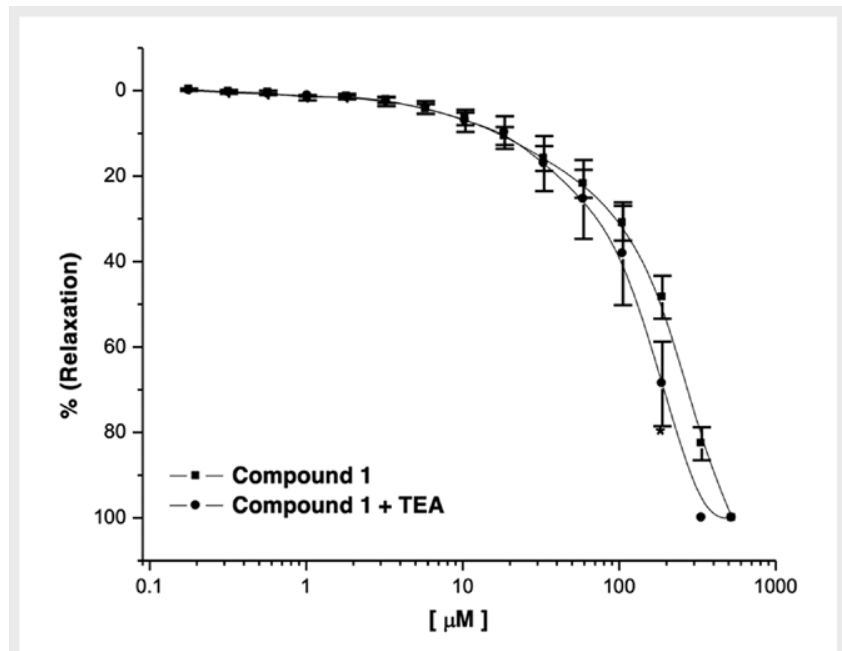

- Fig. 5 Effect of compound $\mathbf{1}$ in the presence and absence of TEA $(5 \mu \mathrm{M})$ on endothelium-intact aorta rings ( ${ }^{*} \mathrm{p}<0.05 \mathrm{vs}$. control). sults might be explained by the presence of a lipid molecule near the nifedipine molecule on 6JP5 structure that was not included on this molecular docking study. As noted from $>$ Table 1, nifedipine and compound $\mathbf{1}$ were found on the same position with the exception of nifedipine and 6JP5 ( $\triangleright$ Figs. 6a and b). On all these experiments, compound $\mathbf{1}$ had lower binding affinity energies than nifedipine. These results may indicate that compound $\mathbf{1}$ may bind to Ltype calcium channel with a higher affinity than nifedipine. Interaction maps analysis ( $\mathbf{F i g} \mathbf{6}$ ) between compound $\mathbf{1}$ and calcium channels reveals that it is possible to observe two possible binding sites, one of them (binding site 1) only observed on 6JPA involving the following residues: T290, M291, E292, F323, L326, T612, G613, E614, D615, W616, F645, T1012, F1013, E1014, A1320, T1321, G1322, E1323, A1324, W1325 and Y1365; and the other binding site (binding site 2) was found on 6JPB, 6JP5 and 6JP8 with the following shared residues on these structures: 1928, 1931, V932, T935, T936, F1008, S1011, T1012, I1052, M1057, F1060, Y1365, M1366 and A1369. Compound 1 had unique interactions on 6JP5 also on binding site 2 with the following residues: V1061, L1367, C1368 and L1371.

As noted on $\mathbf{F i g . ~} \mathbf{7}$, on binding site 1 compound $\mathbf{1}$ was the only one with interactions with residues charged negatively (E292, E614, D615, E1014 and E1323). All negative residues are part of the selective pore $>$ Fig. $\mathbf{6 c}$. On binding site 2 , compound $\mathbf{1}$ made mostly hydrophobic interactions with 6JPB, 6JP5 and 6JP8 with only five hydrophilic residues (T935, T936, Q939, S1011, T1012). Based on binding affinity energies, binding site 2 provides better interactions (mostly hydrophobic) for compound 1 than binding site 1 .

\section{In vivo acute antihypertensive and toxicological assays}

To corroborate the potential efficacy and safety of compound $\mathbf{1}$ as antihypertensive drug, at least in preclinical studies, its antihypertensive action was explored on in vivo SHR, and its safety following OECD toxicological guidelines in acute experiments. Thus, oral administration of compound $\mathbf{1}(50 \mathrm{mg} / \mathrm{kg})$ on SHR significantly reduced systolic ( $\triangleright$ Fig. $\mathbf{8 b}$ ) and diastolic ( $\triangleright$ Fig. $\mathbf{8 c}$ ) blood pressure compared to zero (before administration), and to control group (DMSO $10 \%$ diluted in drinking water), starting 1-3 h after treatment and kept during all experiment. Moreover, $\mathbf{1}$ action is almost 
> Table 1 Molecular docking binding affinities (kcal/mol) of Nifedipine and compound 1 on L-type calcium channel.

\begin{tabular}{|l|l|l|l|l|}
\hline & 6JPA & 6JPB & 6JP5 & 6JP8 \\
\hline Nifedipine & $-5.90+/-0.02(100)$ & $-6.79+/-0.04(98)$ & $-6.86+/-0.14(54)$ & $-7.50+/-0.03(98)$ \\
\hline Compound 1 & $-6.57+/-0.05(99)$ & $-7.80+/-0.00(100)$ & $-7.30+/-0.02(84)$ & $-7.80+/-0.00(100)$ \\
\hline
\end{tabular}

${ }^{*}$ Numbers on parenthesis are the percentage of experiments used to obtain the average binding affinity. One representative conformation of those experiments was used for interaction maps and molecular images.

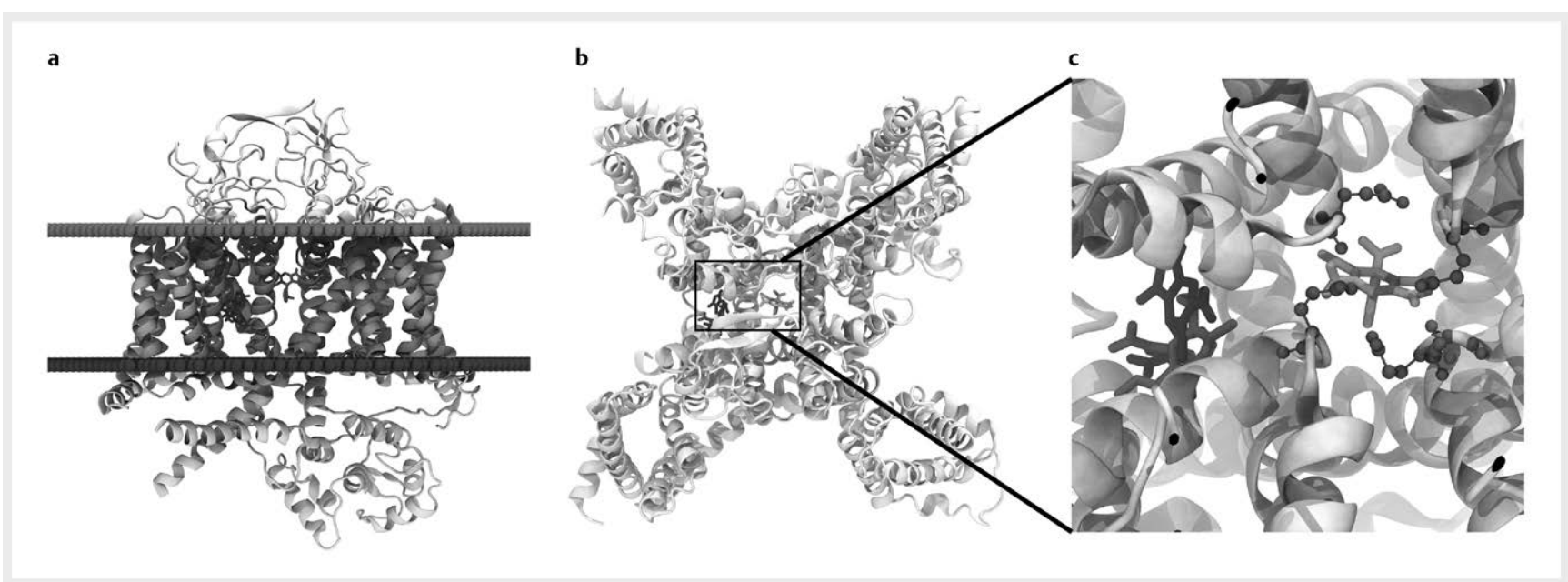

- Fig. 6 Binding sites found for Compound 1. a) 6JP5 Voltage-dependent L-type calcium channel is shown in cartoon representation. Compound 1 and Nifedipine are at channel center on sticks. Top spheres indicate extracellular side on a eukaryotic plasma membrane and low spheres indicate intracellular side according to Orientation of Proteins in Membrane (OPM) database [29]. b) Extracellular view of a). c) Close up of binding sites found for Compound 1 (structure at channel center) with E292, E614, D615, E1014 and E1323 (on ball and sticks representation). Images created using VMD [18].

\section{Nifedipine}

\section{Compound 1}

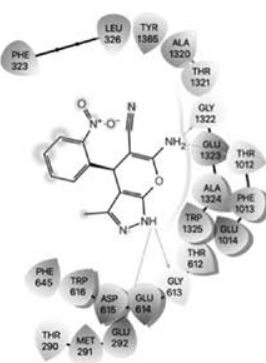

6JPA

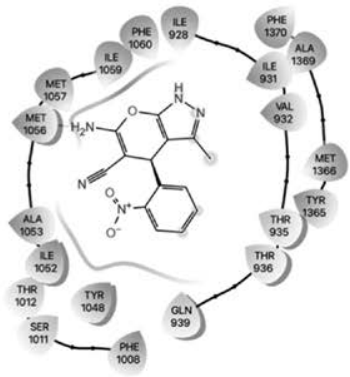

6JPB
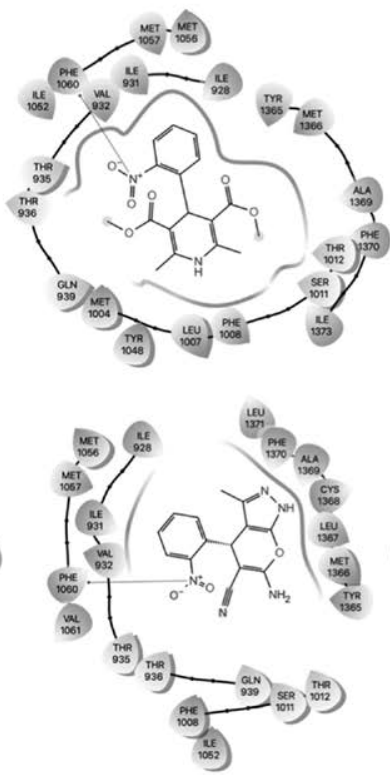

6JP5

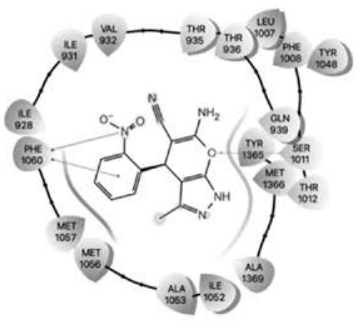

6JP8

- Fig. 7 Interaction maps obtained for Nifedipine and Compound 1. Interaction map for Nifedipine shown only with its original receptor, 6JP5. Compound 1 was found on two possible sites, binding site 1 shown on 6JPA map and binding site 2 shown on 6JPB, 6JP5 and 6JP8. Interaction maps made with Maestro from Schrodinger [17]. 

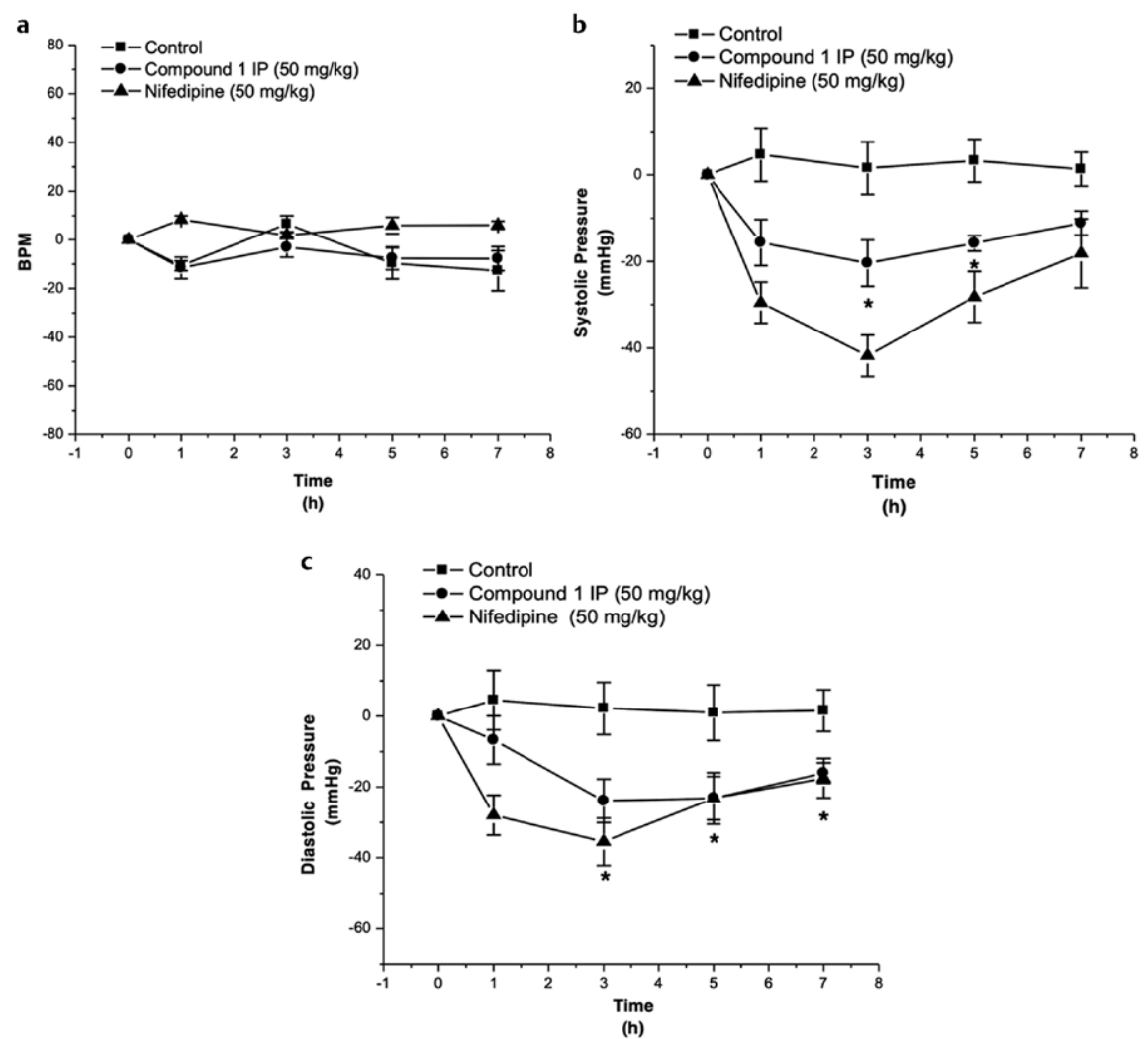

- Fig. 8 Acute effect of compound $1(50 \mathrm{mg} / \mathrm{kg})$ and nifedipine $(50 \mathrm{mg} / \mathrm{kg})$ on a) heart rate, b) systolic and c) diastolic blood pressure in spontaneously hypertensive rats. Results are expressed as the mean of 6 experiments ( ${ }^{*} p<0.05$ vs. control).

equivalent than that displayed by nifedipine $(50 \mathrm{mg} / \mathrm{kg})$, used as positive control. Although nifedipine was more effective at 1 and $3 \mathrm{~h}$ after treatment compared to test sample $\mathbf{1}$. It is worth to mention that nifedipine relatively high dose $(50 \mathrm{mg} / \mathrm{kg}$, i.g.), was used due to the hepatic and extra-hepatic metabolism of the drug [27]; this metabolism might explain the lower in vitro potency of $\mathbf{1}$ vs its similar in vivo potency compared to nifedipine, indicating that in vivo more pathways are involved in drug action. On the other hand, the heart rate did not change, so no positive chronotropic neither inotropic effects were observed ( $\vee$ Fig. 8 a). Results suggest that compound $\mathbf{1}$ is capable to control hypertension in SHR rats mainly by vasorelaxant action, and this effect remains, at least, throughout the experiment showing potential for its development as antihypertensive agent $[2,28]$.

The acute toxicity test carried out based on the OECD protocols, showed that compound 1 evaluated at doses of 5, 50, 300 and 2000 $\mathrm{mg} / \mathrm{kg}$ produced no deaths allowed to determine $\mathrm{LD}_{50}>2000 \mathrm{mg} /$ $\mathrm{kg}$. During the observation period, there were no changes in behavior or apparent toxic reactions. Thus, these results allow to classify the compound evaluated in category 4 according to the GHS [13].

\section{Conclusion}

Compound 1 induced significant antihypertensive activity similar to nifedipine, the mechanism of action is related with vasorelaxant activity linked to a direct L-type calcium channel blockade.

\section{Funding Statement}

This study was supported by Consejo Nacional de Ciencia y Tecnología (CONACyT) Proyecto de Ciencia Básica A1-S-13540. P. Rendón-Vallejo is grateful with CONACyT for the Ph.D. fellowship grant.

\section{Acknowledgments}

The authors thankfully acknowledge the computer resources provided by the Protein Dynamics Laboratory (UAEM). Also, we are indebted with C.V. Rivera-Cerecedo, H.A. Malagón-Rivero and G.X. Ayala-Méndez from the Cell Physiology Institute, UNAM and L.O. Flores-Sánchez, T.E. Villamar-Duque, and F. Barrón-Moreno from FES-Iztacala, UNAM for animal facilities.

\section{Conflict of Interest}




\section{References}

[1] World Health Organization. Hypertension, 2021. (Accessed 10 July 2021)

[2] Schiffrin EL. Circulatory therapeutics: use of antihypertensive agents and their effects on the vasculature. J Cell Mol Med 2010; 14: 1018-1029

[3] Zhou K, Wang X-M, Zhao Y-Z et al. Synthesis and antihypertensive activity evaluation in spontaneously hypertensive rats of nitrendipine analogues. Med Chem Res 2011; 20: 1325-1330

[4] Rendón-Vallejo P, Estrada-Soto S, Vázquez MA et al. Design, synthesis and ex vivo study of the vasorelaxant activity induced by isosteric derivatives of dihydropyridines ( $\mathrm{NH} \rightarrow \mathrm{O}$ ). Lett Drug Des Discov 2016; 13: 353-359

[5] Hernández-Abreu O, Castillo-España P, León-Rivera I, Ibarra-Barajas M, Villalobos-Molina R, Gonzalez-Christen J, Vergara-Galicia J, EstradaSoto $S$. Antihypertensive and vasorelaxant effects of tilianin isolated from Agastache mexicana are mediated by NO/cGMP pathway and potassium channel opening. Biochemical Pharmacology 2009; 78: $54-61$

[6] Vergara-Galicia J, Ortiz-Andrade R, Castillo-España P et al. Antihypertensive and vasorelaxant activities of Laelia autumnalis are mainly through calcium channel blockade. Vasc Pharmacol 2008; 49: 26-31

[7] Rendón-Vallejo P, Hernández-Abreu O, Vergara-Galicia J et al. Ex vivo study of the vasorelaxant activity induced by phenanthrene derivatives isolated from Maxillaria densa. J Nat Prod 2012; 75: 2241-2245

[8] Estrada-Soto S, Rivera-Leyva JC, Ramírez-Espinosa J] et al. Vasorelaxant effect of Valeriana edulis subsp. procera (Valerianacea) and its mode of action as calcium channel blocker. J Pharm Pharmacol 2010; 62: 1167-1174

[9] Vergara-Galicia J, Ortiz-Andrade R, Rivera-Leyva JC et al. Vasorelaxant and antihypertensive effects of methanolic extract from roots of Laelia anceps are mediated by calcium channel antagonism. Fitoterapia 2010; 81: 350-357

[10] Estrada-Soto S, González-Trujano ME, Rendón-Vallejo P et al. Antihypertensive and vasorelaxant mode of action of the ethanolsoluble extract from Tagetes lucida Cav. aerial parts and its main bioactive metabolites. J Ethnopharmacol 2021; 266: 113399

[11] Arias-Durán L, Estrada-Soto S, Hernández-Morales M et al. Antihypertensive and vasorelaxant effect of leucodin and achillin isolated from Achillea millefolium through calcium channel blockade and NO production: functional ex vivo and in silico studies. J Ethnopharmacol 2021; 273: 113948

[12] Hernández-Abreu O, Torres-Piedra M, Garcla Jimenez S et al. Dose-dependent antihypertensive determination and toxicological studies of tilianin isolated from Agastache mexicana. J Ethnopharmacol 2013; 146: 187-191
[13] A Guide to The Globally Harmonized System of Classification and Labeling of Chemicals (GHS). Fourth revised Edition, United Nations, 2011

[14] Berman HM, Westbrook J, Feng Z et al. The protein data bank. Nucleic Acids Res 2000; 28: 235-242

[15] Zhao Y, Huang G, Wu J et al. Molecular basis for ligand modulation of a mammalian voltage-gated $\mathrm{Ca}^{2+}$ channel. Cell 2019; 177: 1495-1506. E12

[16] Trott O, Olson AJ. AutoDock Vina: Improving the speed and accuracy of docking with a new scoring function, efficient optimization, and multithreading. J Comput Chem 2010; 31: 455-461

[17] Schrödinger LLC. Maestro. Schrödinger Release 2021-2. New York, NY

[18] Humphrey W, Dalke A, Schulten K. VMD: visual molecular dynamics. ] Mol Graph 1996; 14: 33-38

[19] Tykocki NR, Boerman EM, Jackson WF. Smooth muscle ion channels and regulation of vascular tone in resistance arteries and arterioles. Compr Physiol 2017; 7: 485-581

[20] Flores-Flores A, Hernández-Abreu O, Rios MY et al. Vasorelaxant mode of action of dichloromethane-soluble extract from Agastache mexicana and its main bioactive compounds. Pharm Biol 2016; 54: 2807-2813

[21] Dolphin AC. Voltage-gated calcium channels: Their discovery, function and importance as drug targets. Brain Neurosci Adv 2018; 2: 1-8

[22] Zamponi GW, Striessnig J, Koschak A et al. The physiology, pathology, and pharmacology of voltage-gated calcium channels and their future therapeutic potential. Pharmacol Rev 2015; 67: 821-870

[23] Hernández F, Sánchez A, Rendón-Vallejo P et al. Synthesis, ex vivo and in silico studies of 3-cyano-2-pyridone derivatives with vasorelaxant activity. Eur J Med Chem 2013; 70: 669-676

[24] Gao S, Yan N. Structural basis of the modulation of the voltage-gated calcium ion channel Cav1.1 by dihydropyridine compounds. Angew. Chem Int Ed Engl 2021; 60: 3131-3137

[25] Fan JS, Palade P. Effects of FPL 64176 on $\mathrm{Ca}^{2+}$ transients in voltageclamped rat ventricular myocytes. Br J Pharmacol 2002; 135 : 1495-1504

[26] Marom M, Hagalili Y, Sebag A et al. Conformational changes induced in voltage-gated calcium channel Cav1.2 by BayK 8644 or FPL64176 modify the kinetics of secretion independently of $\mathrm{Ca}^{2+}$ influx. J Biol Chem 2010; 285: 6996-7005

[27] Grundy JS, Eliot LA, Foster RT. Extrahepatic first-pass metabolism of nifedipine in the rat. Biopharm Drug Dispos 1997; 18: 509-522

[28] Katzung BG. Basic and Clinical Pharmacology: Section III, Antihypertensive Drugs. 14th Edition. McGraw Hill; New York: 2018

[29] Lomize MA, Pogozheva ID, Joo H, Mosberg HI, Lomize AL. OPM database and PPM web server: resources for positioning of proteins in membranes. Nucleic Acids Research 2012; 40: D370-D376 